\title{
Research on standard block classification method of ship interim products based on fuzzy cluster analysis
}

\author{
Xi Li-yang, Wang yue, Zhang hai-yong, Liu Hao-ran, Ji Yong-jun, Liu Jian-feng
}

\begin{abstract}
In order to improve the standardization level of ship block manufacturing process, realize the standardization of block assembly process and working hours, and promote the development of digital hull block manufacturing, in this paper, hull blocks are taken as the research object of classification and grouping, and a fuzzy clustering analysis model is established by using the method of fuzzy clustering analysis. By inputting the production data information of the block process, the quantitative classification of hull blocks is realized. The results show that application of fuzzy cluster analysis to the formation of block families in accordance with the actual process requirements.
\end{abstract}

Index Terms - interim product, fuzzy cluster analysis, standard block families

\section{INTRODUCTION}

With the increase of competition in the shipbuilding industry and the increase of production costs, it is particularly important to promote the standardized lean management of the hull production process. To achieve standardization of the production process, it is necessary to classify the interim products of the hull and the grouping of interim products. The rationality directly affects the level of standardized lean management.

In the actual shipbuilding process, the classification of the hull block type mostly stays in the qualitative analysis, and the interim products are classified according to experience. When the number of interim products in the classification is large, the classification of the blocks by different designers may vary widely. There is no guarantee that the classification results will be reasonable in the shipbuilding process. Therefore, the hull block classification of the interim products requires the introduction of mathematical methods for quantitative analysis. Input the relevant data of process features, and output the corresponding classification information of intermediate products after the analysis of fuzzy clustering algorithm.

Xi Liyang, School of Naval Architecture \& Ocean Engineering, Jiangsu University of Science and Technology, Zhenjiang, Jiangsu, China 18796089106

Wang yue, School of Naval Architecture \& Ocean Engineering, Jiangsu University of Science and Technology, Zhenjiang, Jiangsu, China, 13952807905 .

Zhang hai-yong, Shanghai Waigaoqiao Shipbuilding Co. Ltd., Shanghai, China, 1838886746

Liu Hao-ran, School of Naval Architecture \& Ocean Engineering, Jiangsu University of Science and Technology, Zhenjiang, Jiangsu, China 15190878056

Ji Yong-jun, Shanghai Jiao Tong University ,Shanghai,China, 15190878056

Liu Jian-feng, Shanghai Waigaoqiao Shipbuilding Co. Ltd., Shanghai, China, 13006163874
There are three methods for classifying interim products: visual inspection, production process analysis, and coding classification. Storch, Richard Lee established the CBG (Common Block Generic) based on the principle of mass customization, block complex matrix and clustering method, and described the overall framework of this theory, and proposed an effective classification group for interim products. Method ${ }^{[1]}$; Zhong Hong-cai based on the classification group theory by analyzing the manufacturing features of the parts, the whole ship parts are divided into five processing families ${ }^{[2]}$, Zhong Hong-cai also proposed the Parks distance method to similar features method of clustering block components ${ }^{[3]}$; Wang Feng-xiang uses hull block as an interim product to study the classification of ship block interim products based on the geometric shape and area features of hull block ${ }^{[4]}$; According to the technical features and economic features, the ship's intermediate products are analyzed according to the technical features and economic features, and the ship segmentation is classified into fuzzy classification $^{[5]}$; Feng Guo-qing proposes an application of fuzzy cluster analysis method and numerical simulation technology to optimize cluster analysis and evaluation method $^{[6]}$; By analyzing the relationship between similarity and average number of parts in the same group, Lu Kai-Jiang proposed a fuzzy clustering analysis method based on classification and coding of mechanical parts ${ }^{[7]}$.

\section{FEASIBILITY ANALYSIS OF FUZZY CLUSTERING FOR PARTITION TYPE ANALYSIS}

"Fuzzy Clustering Analysis" is a quantitative clustering method using the language of Fuzzy Mathematics. Clustering refers to the process of distinguishing and classifying things according to certain requirements and rules. In this process, there is no prior knowledge about classification, and only the similarity between things is used as the criterion of generic planning. Cluster analysis refers to the method of using mathematical methods to analyze the classification of individual objects according to the features of things themselves. Cluster analysis belongs to multivariate statistical analysis. It divides a set of samples without class labels into several subsets according to some rules, and makes the samples of similar individuals be divided into different categories as far as possible. Traditional clustering analysis is a kind of hard partition. This partition method strictly divides each individual object to be identified into a certain class. However, in fact, there is no strict attribute distinction between most objects, and there is intermediary in the aspect of character and category. At this time, fuzzy clustering analysis is needed ${ }^{[8]}$.

Because there are a lot of process information involved in the 
hull production process, assembly process is complex, and the types of segments that meet the production process requirements are divided. The influencing factors are multi-dimensional, and each influencing factor affects each other. In this paper, the method of fuzzy clustering analysis is used to classify the block samples based on the actual data.

\section{HULL BLOCK FEATURE INFORMATION}

After the hull is decomposed according to the principle of sectional division, a number of sectional interim products are formed as shown in Figure 1. In order to simplify the research object and form a standard block assembly process information model, it is necessary to classify the block types by referring to a variety of classification elements ${ }^{[9]}$.

In order to make the block type have practical production significance, the blocks are classified into groups according to the type of problem. The main clustering reference elements are the block area, geometric features and operation features. Block area mainly refers to the spatial location information of the bock, which is represented by the code bit of the block coding area[10]. Geometric features mainly express the block shape features, determine the choice of the construction method of the block, and use the proportion of the surface as the classification reference coefficient. The operation features mainly include the classification and evaluation of factors such as block assembly information and jig information in the production process. The assembly information indicates that the plane workshop is 1 , the surface workshop is 2 , the field area is 3 , the jig information indicates that the plane jig is 1 , the single jig is 2 , the hyperbolic jig is 3 , and the jig is 4 . The above parameters are expressed as follows:

1) block area is $u_{1}$;

2) block geometric feature is $u_{2}$;

3) Operation features include sblock assembly workshop information $\mathrm{u}_{3}$ and jig information $\mathrm{u} 4$.

Establishing block fuzzy clustering function:

$$
\mathrm{f}=\left(\mathrm{u}_{1}, \mathrm{u}_{2}, \mathrm{u}_{3}, \mathrm{u}_{4}\right)
$$

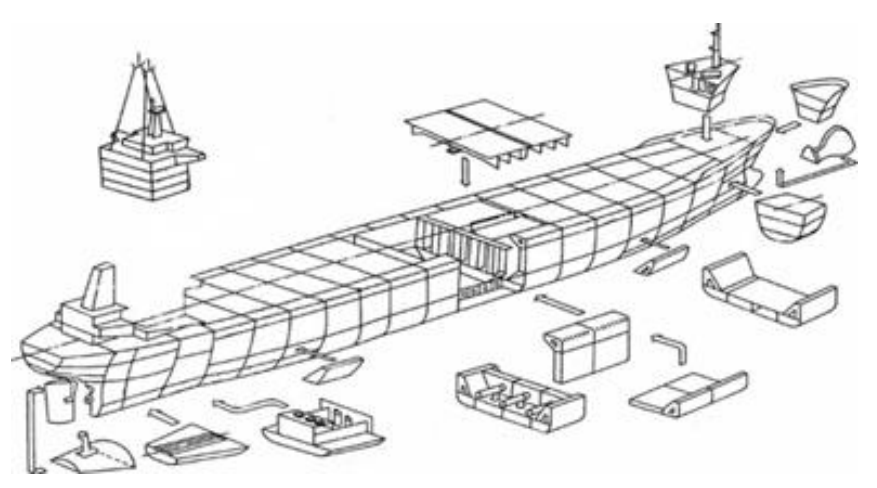

Fig.1 Standard block information diagram

\section{USING FUZZY CLUSTERING ANALYSIS TO CLASSIFY AND GROUP BLOCKS}

\section{A. General steps of cluster analysis}

Fuzzy clustering analysis is a method to classify objects quantitatively on the basis of fuzzy similarity matrix. The following steps are introduced to classify objects quantitatively by using transitive closure of fuzzy clustering. Let the set of classified objects be $\mathbf{X}=\left\{\mathrm{x}_{1}, \mathrm{x}_{2}, \cdots, \mathrm{x}_{\mathrm{m}}\right\}$, each object $x_{i}$ has $m$ feature indicators, $x_{i}$ can be expressed by m-dimensional feature indices vector: $x_{i}=\left(x_{i 1}, x_{i 2}\right.$, , $\mathrm{x}_{\mathrm{im}}$ ) , $i=1,2, \cdots, \mathrm{n}$; where $\mathrm{x}_{\mathrm{ij}}$ represents the $\mathrm{jth}$ feature index of the ith object. Then all the feature indexes of $n$ objects form a feature index matrix of $X$, which is recorded as $\mathbf{X}^{*}=\left(\mathrm{x}_{\mathrm{ij}}\right)_{\mathrm{n} * \mathrm{~m}}$.

$$
X^{*}=\left(\begin{array}{cccc}
x_{11} & x_{12} & \cdots & x_{1 m} \\
x_{21} & x_{22} & \cdots & x_{2 m} \\
\cdots & \cdots & \cdots & \cdots \\
x_{n 1} & x_{n 2} & \cdots & x_{n m}
\end{array}\right)
$$

\section{(1) Data standardization}

Since the dimension and magnitude of $\mathrm{m}$ characteristic indices are not necessarily the same, it is possible to highlight the role of feature indices with very large magnitude in classification in the process of calculation, thus reducing or even excluding the role of feature indices with very small magnitude. Data standardization enables each index value to be unified in a common range of numerical features. The first variable is optimized. After $\mathrm{x}_{\mathrm{ij}}$ is transformed into $\mathrm{x}_{\mathrm{ij}}$, the data interval is in $[0,1]$.

\section{(2) Establishment of fuzzy similarity matrix}

Clustering is to identify the similarity degree of elements in X set according to some criteria, and classify the similar element objects into one group. Therefore, the number $r_{i j}$ in $[0,1]$ is used to express the similarity degree of elements $x i$ and $x_{j}$ in $X$.Let the normalized similarity coefficient between $\mathrm{x}_{\mathrm{i}}=\left(\mathrm{x}_{\mathrm{i} 1}\right.$, $\left.\mathrm{x}_{\mathrm{i} 2}, \cdots, \mathrm{x}_{\mathrm{im}}\right)$ and $\mathrm{x}_{\mathrm{j}}=\left(\mathrm{x}_{\mathrm{j} 1}, \mathrm{x}_{\mathrm{j} 2}, \cdots, \mathrm{x}_{\mathrm{jm}}\right)$ be $\mathrm{r}_{\mathrm{ij}} \in[0,1]$, and then the fuzzy similarity matrix $\mathbf{R}=\left(r_{\mathrm{ij}}\right)_{\mathrm{m} \times \mathrm{n}}$ between elements is obtained.

\section{(3) Fuzzy clustering}

The fuzzy relation matrix between objects is constructed by the above method. Different classifications can be obtained for different $\lambda \in[0,1]$. The appropriate confidence level value $\lambda$ is selected, and the dynamic clustering is carried out according to the $\lambda$-matrix $\overline{\boldsymbol{R}}_{\lambda}$. Finally, the dynamic clustering graph is generated.

The flow chart of the fuzzy clustering analysis is shown in Figure 2.

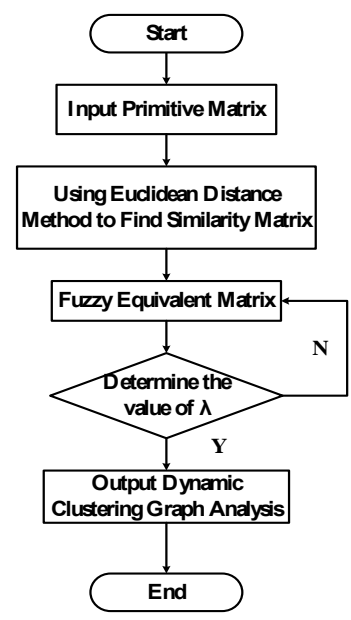

Fig.2 Flow diagram of fuzzy clustering 


\section{B. Application of cluster analysis}

Taking a 270,000-ton bulk carrier as an example and 232 block elements of the main hull as the research object, the matrix relationship between subsection coding and feature parameters is established as shown in Table 1.

Table 1 Original data for clustering(part)

\begin{tabular}{cccccc}
\hline NO. & $\begin{array}{c}\text { BLOCK } \\
\text { NO. }\end{array}$ & $\mathrm{u}_{1}$ & $\mathrm{u}_{2}$ & $\mathrm{u}_{3}$ & $\mathrm{u}_{4}$ \\
\hline 1 & 101 & 1 & 0.92 & 2 & 3 \\
2 & 103 & 1 & 0 & 2 & 1 \\
3 & 123 & 1 & 0.46 & 2 & 1 \\
4 & 161 & 1 & 0.73 & 2 & 3 \\
5 & 203 & 2 & 0 & 1 & 1 \\
6 & 221 & 2 & 0.54 & 1 & 1 \\
7 & 224 & 2 & 0.31 & 1 & 1 \\
8 & 261 & 2 & 0.76 & 2 & 3 \\
9 & 265 & 2 & 0 & 1 & 1 \\
10 & 521 & 5 & 0 & 1 & 1 \\
\hline
\end{tabular}

In this paper, the sample data are analyzed and clustered in the environment of MATLAB software.

\section{(1) Data standardization}

Each data of the fuzzy local matrix is compressed to [0,1], and the range normalization method is used to standardize the data.

$$
x_{i j}{ }^{\prime}=\frac{x_{i j}-\min _{1 \leq i \leq n}\left\{x_{i j}\right\}}{\max _{1 \leq i \leq n}\left\{x_{i j}\right\}-\min _{1 \leq i \leq n}\left\{x_{i j}\right\}}
$$

The normalization matrix of some original data can be obtained by using MATLAB operation as follows:

$$
X=\left(\begin{array}{cccc}
0.7924 & 1 & 0.5275 & 0.6005 \\
0.7924 & 1 & 0.6547 & 0.0865 \\
0.7924 & 1 & 0.6547 & 0.2570 \\
0.7924 & 1 & 0.5275 & 0.0456 \\
0.0880 & 1 & 0.6547 & 0.0865 \\
0.0880 & 1 & 0.6547 & 0.4907 \\
0.0880 & 1 & 0.6547 & 0.1811 \\
0.0880 & 1 & 0.5275 & 0.1332 \\
0.0880 & 1 & 0.6547 & 0.0865 \\
0.7294 & 1 & 0.6547 & 0.0865 \\
\square & \square \square & \square & \square
\end{array}\right)
$$

(2)For the above standardized matrices, the direct Euclidean distance method is used to calculate and analyze the differences between samples. The distance matrices of some sample data are as follows:

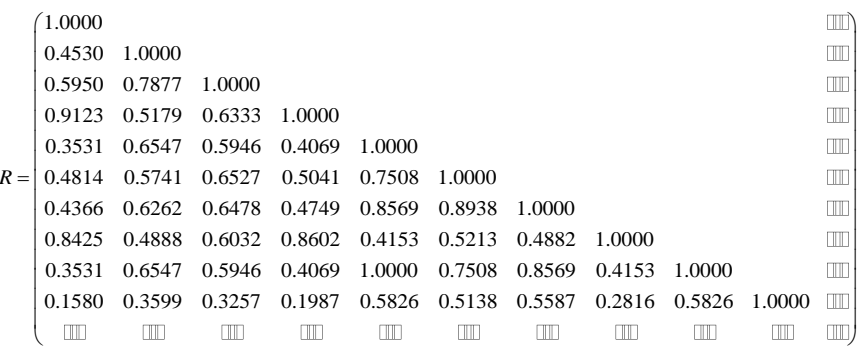

(3) Transfer closure method is used to transform the above-mentioned fuzzy matrix into a fuzzy equivalent matrix. According to the theorem, the transfer closure can be obtained by quadratic method, that is, the fuzzy equivalent matrix can be obtained, and then the dynamic clustering pedigree graph can be formed by changing the value of lambda from large to small. As shown in Figure 3, different classifications can be obtained by choosing the appropriate confidence level value lambda.

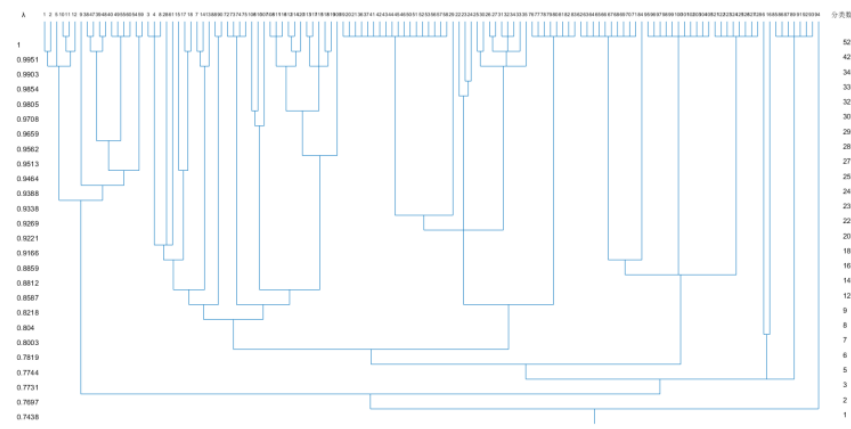

According to the above analysis and comprehensive comparison, select $\lambda=0.8754$ and the number of classifications is 17 , and then the subsection classification of 270,000 tons bulk carriers can be obtained as shown in the figure 4 below.

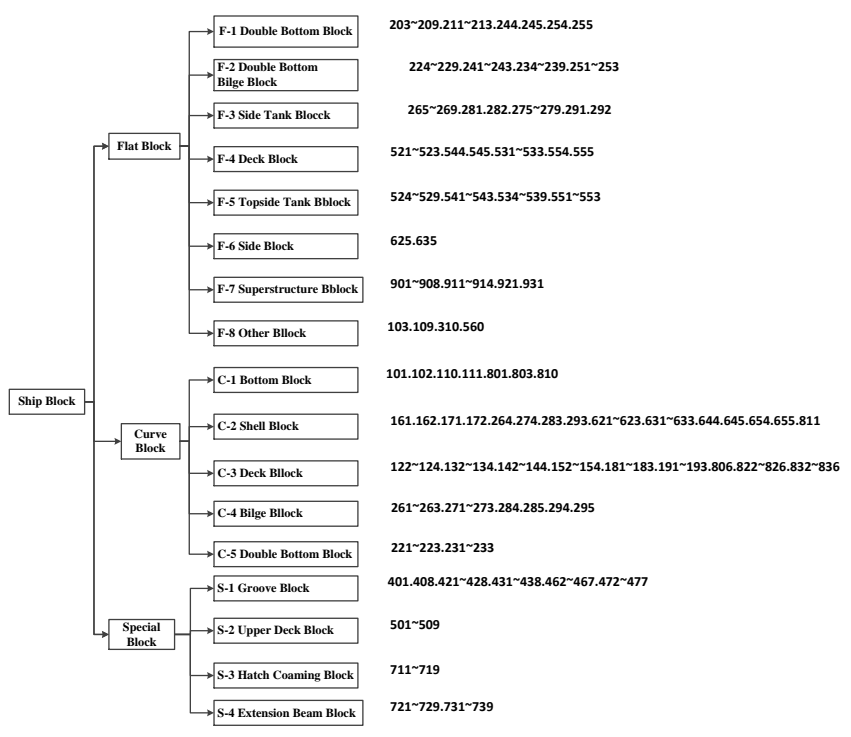

\section{CONCLUTION}

1. The method of fuzzy clustering analysis is applied to the classification of hull block families, and the model of hull block clustering analysis is established. Compared with the classification method based on artificial reasoning, the result of fuzzy clustering analysis can better meet the needs of process requirements. 
2. - The hull block clustering analysis model is a multi-input and single-output fuzzy classification model, which is not only suitable for the block clustering analysis, but also has certain universal application significance for other classification problems similar to multi-factor input and single-output.

3) In view of the classification of hull blocks, it is feasible to use the fuzzy clustering analysis method to cluster the hull blocks.

\section{REFERENCES}

[1] Storch, Richard Lee. Theoretical foundation for common generic block in assembly production [D]. University of Washington, 2000.

[2] Zhong Hong-cai,Jiang Ru-hong and Tan Jia-hua. Research on Formation of Interim Product Family in Shipbuilding[J]. SHIP ENGINEERING, 2003(3):51-61.

[3] Zhong Hong-cai, Liu Jian-feng, Tan Jia-hua. Interim products clustering method in shipbuilding[J]. Journal of Shanghai Jiaotong University, 2003(8):1234-1237.

[4] Wang Fengxiang. Study and application of palletized lane benchmarkof section assembly based on lean shipbuilding[D]. Jiangsu University of Science and Technology,2014.

[5] YU Feng-ping, XIAO Hong-jun. Evaluation on the intermediate product family classification standards based on fuzzy pattern identification: taking the example of assembly of shipbuilding[J]. SHIP SCIENCE AND TECHNOLOGY. 2011(4):140-143.

[6] Feng Guo-qing, Pan Li-yan, Kong Bing, Luo Jia-shun. Hierarchical optimization research based on fuzzy clustering analysis[J].Reservoir evaluation and development, 2018(3): 30-34.

[7] Lu Kai-jiang, Zhang Ning, Yang Feng.Analysis of fuzzy sets and systems based on the classified coding for parts[J]. Light Industry Machinery,2003(3):7-9.

[8] Xi Li-yang, JIANG Zhi-yong, Liu Jian-feng. Research on Man-hour Quota Model of Hull Block Working Package Based on PSO-BP Neural Network[J].Ship Engineering,2019(4):50-56.

[9] Xi Li-yang.Research and Application on Optimization of the Standard Sequence Engineering Plan Management Technology Oriented to Lean Production[D]. Jiangsu University of Science and Technology,2019.

[10] Rao zhi-gang.The Application of fuzzy clustering analysis to the ship block fitting[D]. Wuhan University of Technology.2006.

Xi Liyang (1991 -), male, master's degree student, Mainly engaged in research on the direction of ship manufacturing process control. 